\title{
Postoperative Cognitive Dysfunction and the Protective Effects of Enriched Environment: A Systematic Review
}

\author{
Momin $\mathrm{Hua}^{\mathrm{a}} \quad \mathrm{Jia} \mathrm{Min}^{\mathrm{b}}$ \\ ${ }^{a}$ First Clinical Medical College, Nanchang University, Nanchang, PR China; ${ }^{b}$ Department of Anesthesiology, \\ First Affiliated Hospital of Nanchang University, Nanchang, PR China
}

\section{Keywords}

Postoperative cognitive dysfunction · Enriched

environment · Neuroinflammation · Alzheimer's disease

\begin{abstract}
Background: Currently, the number of individuals who undergo surgery is greatly increased. As a consequence, postoperative cognitive dysfunction (POCD) has gradually gained more attention. Summary: POCD is a perioperative complication requiring sensitive preoperative and postoperative neuropsychiatric tests, and its incidence in both cardiac and noncardiac surgery is high, especially in elderly individuals. Surgical, patient, and anesthetic factors may all lead to the occurrence and development of POCD. The key mechanism of POCD may be the inflammatory response of the central nervous system during surgery, which is similar to that of Alzheimer's disease (AD). Enriched environment $(E E)$, a factor that can significantly improve and prevent neurodegenerative diseases, may have a beneficial effect on POCD. Key Messages: This review aims to elucidate the mechanism of the occurrence and development of POCD, analyze the possible influence of EE on POCD at the molecular level, and provide a direction for its treatment.
\end{abstract}

C 2021 S. Karger AG, Basel

\section{Introduction}

Postoperative neurological complications pose a great threat to human health, especially in elderly patients. Postoperative cognitive dysfunction (POCD) is a syndrome defined by the continuous decline of cognitive performance, including attention, executive function, memory, visual spatial ability, and psychomotor speed, in a series of neuropsychological tests before and after surgery [1]. Globally, it is estimated that $50 \%$ of all older individuals have undergone at least one operation and it is known that changes associated with aging affect an individual's ability to withstand injury, surgery, and anesthetic stress [2]. Thus, POCD has far-reaching social and economic impacts, as cognitive decline is associated with loss of independence, lower quality of life, and death [3]. Age, education level, preoperative cognitive ability, and complications are all considered to be factors affecting the occurrence and development of POCD [2]. Meanwhile, studies have suggested that some preoperative factors may trigger the neuropathogenesis of Alzheimer's disease $(\mathrm{AD})$ to cause POCD [4]. Enriched environment (EE), which improves behavioral, cellular, and molecular defects in animal models, can provide animals with the optimum conditions to enhance exploration, cognitive activities, social interaction, and physical exercise [5]. This suggests that EE may become a strategy for the prevention and treatment of POCD.

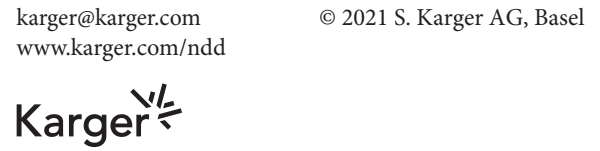




\section{Definition and Incidence of POCD}

The earliest description of POCD was put forward by Bedford [6] in 1955. According to the International Society of Postoperative Cognitive Dysfunction (ISPOCD), POCD is a defect in $\geq 1$ areas of the mental state observed in patients after operation [7]. It is a subtle thought process disorder that can affect different cognitive areas such as verbal memory, language comprehension, visual memory, visual spatial abstraction, attention, and executive function. POCD is defined as a syndrome characterized by a continuous decline in cognitive performance in a series of neuropsychological tests before and after surgery [1].

There are no formal criteria for the evaluation and diagnosis of POCD, which require sensitive preoperative and postoperative neuropsychiatric tests. Therefore, the current incidence of POCD varies greatly according to the statistical definition of the cognitive data applied. Consequently, it is necessary to develop consistent diagnostic criteria for POCD to conduct a more meaningful study of this cognitive dysfunction [3].

Early studies showed that POCD occurs more frequently after cardiac surgery than other types of surgery [8], with the highest incidence of $30-70 \%$ at discharge, followed by $20-30 \% 6$ months after the operation and 15-25\% 12 months after the operation [9]. In 1998, a multinational research team published the first largescale prospective study to describe cognitive decline after noncardiac surgery [7]. Moller et al. [7] studied the risk factors for POCD in 1,218 patients $>65$ years of age who underwent noncardiac surgery, and then compared them with 321 controls who did not undergo surgery but also underwent repeated neuropsychological tests. The study showed that the prevalence of POCD was $26 \%$ 1 week after the operation and dropped to $10 \%$ at 3 months after the operation, and a similar prevalence was found 12 months postoperatively. In the nonoperative controls, a $3 \%$ incidence of POCD was found for each time period. Monk et al. [10] also recorded the presence of POCD in patients discharged from noncardiac surgery. Their research suggested that $36.6 \%$ of surgical patients who developed POCD were 39 years old, $30.4 \%$ were aged between 40 and 59 years, and $41.4 \%$ were $\geq 60$ years. Three months later, $12.7 \%$ of the patients $>60$ years still had POCD. However, recent randomized studies found that the incidence of POCD is similar in cardiac surgeries regardless of whether cardiopulmonary bypass $(\mathrm{CPB})$ is performed [11], which is worthy of further study.

\section{Factors Affecting the Occurrence and Development of POCD}

The main risk factors for POCD include major invasive surgery, the duration of anesthesia, an advanced age, a history of alcoholism, the use of anticholinergic drugs, and other perioperative events. The release of cytokines caused by the systemic stress response induced by anesthesia and surgery may lead to changes in brain function and participate in the development of POCD [12].

\section{Patient Factors}

Advanced age is the highest known risk factor associated with POCD. The ISPOCD1 study analyzed the risk factors for POCD in patients undergoing noncardiac surgery; it found that, 3 months after the operation, the incidence of POCD was $7 \%$ in patients aged 69 years and $14 \%$ in patients $>69$ years of age [7]. Advanced age is characterized by impaired functions in many regulatory processes, which can lead to increased physical and mental fragility as well as a reduced ability to deal with physical stressors such as anesthesia and surgery. In addition, older individuals are more likely to have a variety of underlying diseases like diabetes, renal insufficiency, or cardiovascular disease [12]. Additionally, elderly patients tend to have more risk factors for neurovascular diseases, more white-matter damage, and lower cognitive reserves [13], all of which increase the risk of perioperative complications.

Some studies have suggested that POCD incidence is lower in patients with a higher level of education than in patients with a lower level of education. In people with a higher level of education, the brain is constantly exposed to challenging psychological activities that may slow the development of dementia by utilizing the cognitive reserves and improving the efficacy of synapses, thus changing the progression of neuronal damage [2]. The duration of a person's educational and vocational activities may increase cognitive reserves, making some people less vulnerable to cognitive changes [14]. Moderate physical activity and social participation also play a positive role in promoting the cognitive function of elderly individuals, and elderly surgical patients who are inactive, depressed, and socially isolated are more likely to suffer cognitive impairment [2].

\section{Perioperative Factors}

The functioning of the central nervous system (CNS) depends on an adequate supply of oxygen and nutrients, effective removal of waste, and the existence of an appro- 


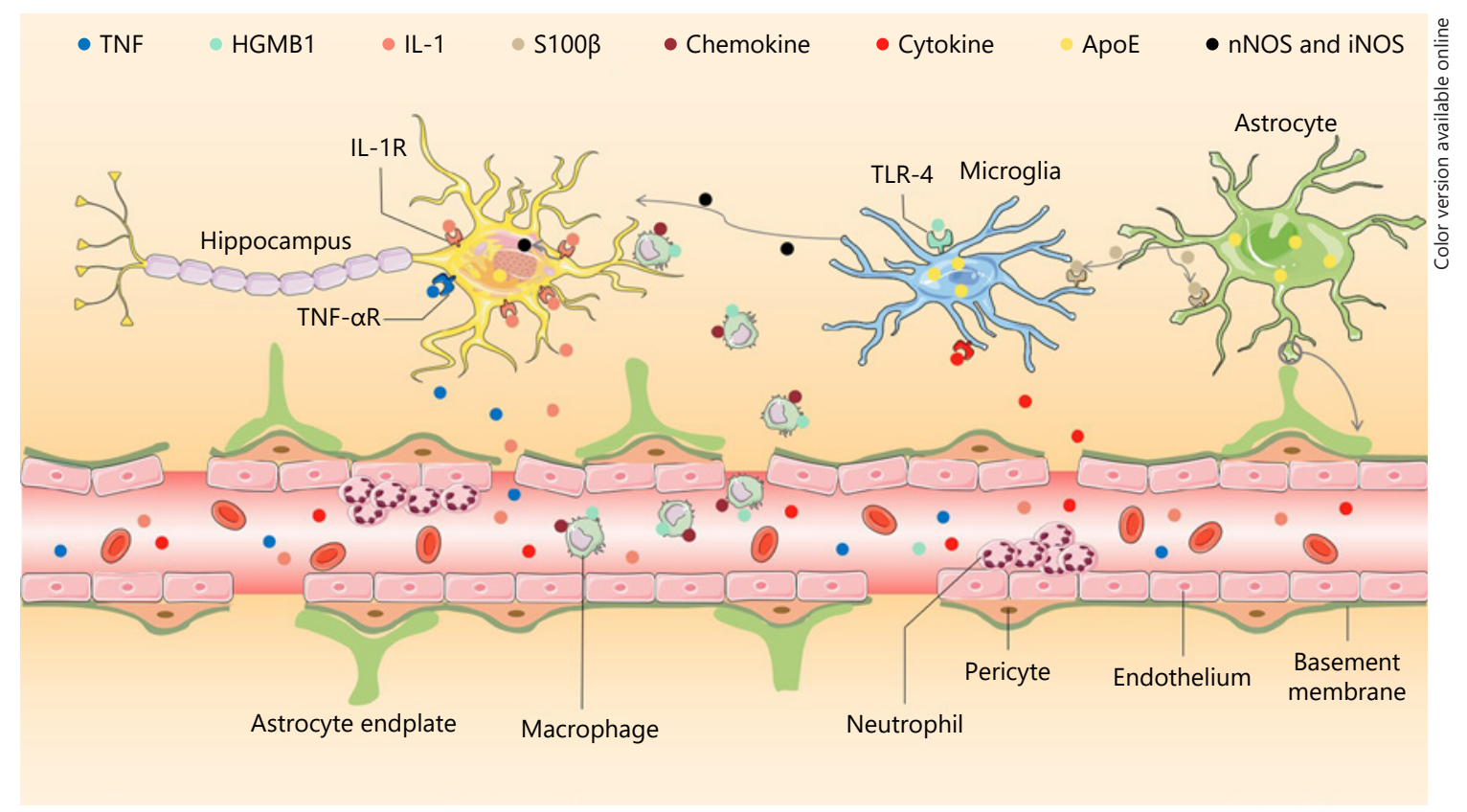

Fig. 1. This picture depicts the CNS immune response caused by peripheral inflammation.

priate neurochemical environment. A metabolic imbalance in the brain from any cause may lead to overall dysfunction, which can lead to a decline in cognitive function after surgery [1]. Wollman and Orkin [15] reported that extreme hypocapnia during anesthesia is associated with prolonged cognitive impairment for at least 6 days after hyperventilation [1]. Obviously, to avoid postoperative cognitive dysfunction, it is essential to provide adequate oxygen to the brain and all the important organs.

Cardiac surgery and certain orthopedic surgeries can lead to a relatively high incidence of POCD. One hypothesis is that cerebral microemboli can lead to the mild neurological impairment associated with POCD [1]. The high incidence of POCD in cardiac patients is attributed to microembolic events that can cause focal cerebral infarction during CPB [12]. CPB can also cause specific activation of the inflammatory response through the immune system, ischemia-reperfusion injury, complement activation, and heparin and protamine neutralization after blood contact with artificial materials during a bypass [16]. In a mouse model, hippocampal-dependent memory impairment induced by orthopedic surgery and anesthesia was found to be associated with an increase in plasma cytokines and the activation of the interleukin (IL)- $1 \beta$ pathway in the hippocampus [17]. Further studies have demonstrated that isoflurane alone activates the IL- $1 \beta$ pathway and leads to hippocampal cell damage, which may lead to related cognitive impairment [18].

Three main factors lead to cognitive impairment after cardiac surgery: embolism, low perfusion, and inflammation. The results of the experiments conducted by Ito et al. [19] show that patients with asymptomatic cerebral ischemia are more likely to develop POCD after cardiac bypass surgery. Studies have shown that there is a relationship between low preoperative levels of the anti-endotoxin core antibody and POCD in patients undergoing cardiac surgery. This suggests that a decreased immune response to endotoxin may be the cause of POCD [2]. Severe systemic inflammation can lead to multiple organ failure, including in the brain, and a variety of clinical consequences such as delirium and septic encephalopathy and ranging from mild cognitive impairment to coma [1].

\section{Pathogenesis of POCD}

\section{Neuroinflammation Mechanism}

The mechanism of POCD has not been fully elucidated, but the most accepted mechanism is neuroinflammation, especially in the hippocampus. Although cognitive processes involve multiple brain regions, the hippocampus, which contains the largest number of cytokine receptors, plays a clear role in learning and mem- 
ory formation. In particular, the hippocampus contains the highest density of IL-1 receptors. Although physiological levels of IL-1 are essential for optimizing memory and learning processes, high levels are associated with decreased cognitive function in animal models. Additionally, the hippocampus appears to be particularly vulnerable to the harmful effects of elevated levels of inflammatory mediators, such as high-mobility group protein 1 (HMGB1), because of the high density of tumor necrosis factor (TNF)- $\alpha$ receptors and many other receptors on the surface of endothelial cells in this region [20].

Through animal experiments, Terrando et al. [21] showed that the surgical immune response plays an important role in POCD. Their studies showed that inflammatory TNF or NF- $\kappa \mathrm{B}$ signaling cascades were activated during peripheral surgery in mice, resulting in the release of cytokines and the destruction of the integrity of the blood-brain barrier (BBB). Consequently, macrophages are more likely to migrate to the hippocampus, and neutrophils are more likely to adhere to capillary endothelial cells, resulting in memory impairment. On the other hand, activating the anti-inflammatory cholinergic signaling cascade to prevent the secretion of proinflammatory cytokines can prevent damage to cognitive function. Moreover, some researchers believe that sedation and anesthesia can lead to limited ischemia of brain endothelial cells and induce hypoxia. Acute hypoxia can induce activated neutrophils to migrate and adhere to endothelial cells, which leads to the development of microthrombi and the release of powerful pressor substances, resulting in complete capillary blockage and blood flow obstruction. Therefore, the response of the CNS to systemic inflammatory mediators can lead to delayed recovery or long-term postoperative CNS dysfunction [1] (Fig. 1).

In different preclinical models of non-CNS surgery, neuroinflammation is often associated with behavioral and memory dysfunction. Previous studies have shown that chemokines and injury-related molecules (e.g., HMGB1) can activate bone marrow-derived macrophages and contribute to overall brain pathology after aseptic trauma [13]. Increased levels of the proinflammatory cytokine IL- $1 \beta$ in the CNS lead to an increase in reactive oxygen species (e.g., nNOS and iNOS) which is coupled with the destruction of long-term potentiation (LTP) [20]. Studies have indicated that nNOS and iNOS increase the production of peroxynitrite, inhibit mitochondrial respiratory enzymes, and damage DNA [22] (Fig. 1).

\section{Activation of Microglia and Astrocytes}

Inflammation of the CNS also depends on the activation of microglia to drive the production of local oxidation products. Microglia express specific receptors for inflammatory signals, such as bacterial endotoxins or endogenous ligand receptors, including the HMGB1 receptor and especially Toll-like receptor (TLR)-4. The increase in peripheral inflammatory factors induced by surgery leads to morphological changes and the upregulation of TLR-4 in microglia [23] (Fig. 1). Surgical stress increases the levels of NADPH oxidases (NOX enzymes) in the brain, and NOX enzymes are key to the regulation of oxidative stress which is related to behavioral changes in mice [24]. NOX enzymes are also involved in the increased oxidative stress observed in a variety of brain diseases ranging from mental illness to neurodegenerative diseases [25].

Astrocytic S100 calcium-binding protein $\beta$ (S100 $\beta)$ is highly abundant in the brain and plays a crucial role in multiple homeostasis- and injury-related processes through autocrine and paracrine signal transduction. S100 $\beta$ can directly induce neuronal injury or activate microglia and astrocytes and induce microglial oxide production [20]. S100 $\beta$-induced neuroinflammation mediates RAGE (advanced glycosylation end product-specific receptor) signaling in microglia. The RAGE signaling pathway may upregulate proinflammatory cytokines through the NF- $\mathrm{KB}$ signaling pathway, indicating that $S 100 \beta$-induced neuroinflammation may play a role in the pathogenesis of surgery-induced cognitive impairment [26] (Fig. 1).

\section{Pathological Changes Similar to AD}

Studies have suggested that POCD and AD have similar pathological changes and serological markers. Histologically, $\mathrm{AD}$ is characterized by neurofibrillary tangles, which consist of pairs of spiral filamentous hyperphosphorylated Tau proteins, and extracellular amyloid $\beta$ plaques. The pathological effects of these changes are increased neuronal death and synaptic loss, mainly in the cholinergic neurons in the basal forebrain. The central cholinergic system plays a critical role in the formation and regulation of consciousness, learning, and memory [27]. Moreover, IL-18 is colocalized with the Tau protein and amyloid $\beta$ plaques, and it mediates the hyperphosphorylation of Tau. IL-18 can affect the integrity of neurons and increase neuroinflammation in the brain, resulting in a decline in cognitive function [26].

Eckenhoff et al. [28] demonstrated that clinical concentrations of halothane and isoflurane enhanced the 
oligomerization and aggregation of amyloid peptides in cell culture. Studies have also indicated that isoflurane activates an enzyme called cysteinase, which may contribute to the formation of neurofibrillary tangles and the production of amyloid $\beta$ protein [29]. The amyloid $\beta 1-42$ oligomer can interfere with insulin signaling, thus impairing cognitive and metabolic processes in the hippocampus [26].

\section{Other Molecular Mechanisms}

The existence of the apolipoprotein E (ApoE) allele is also considered to be a risk factor for the development of POCD. ApoE is not only an important lipid and cholesterol transport molecule but also an immunomodulator that is constitutively expressed in the brain and upregulated after brain injury, and it exists in neurons, oligodendrocytes, microglia and astrocytes [30] (Fig. 1). ApoE has both pro- and anti-inflammatory effects, which may be related to regulating the degree of astrocyte and microglia activation [20]. In addition, IL-1 $\beta$ has been revealed to stimulate the secretion of ApoE in mixed glial cultures [31].

Glutamate signaling is particularly important in longterm potentiation and supports the formation of memory. Experimentally induced peripheral inflammation fundamentally changes the activity of hippocampal neurons, LTP, and synaptic plasticity in rats. Decreased expression of the glutamate receptor subtype GluR2 leads to the enhancement of signal transduction mediated by AMPA and NMDA. Additionally, HMGB1 can enhance glutamate signal transduction through NMDA receptors and aggravate the glutamate-induced toxicity associated with the degradation process [20].

An animal study also suggested that postoperative memory impairment may be due to the anesthesia-induced upregulation of GABA-A receptors containing a5 subunits (which can inhibit LTP) in the hippocampus. Therefore, it can be predicted that the upregulation of these receptors will result in learning and memory disorders which may induce human POCD [32].

\section{The Definition of EE}

The experimental model of Enriched Environment (EE) was first described by Donald Hebb [33] in the context of neuroscience. He compared rats allowed to roam freely in his home with rats housed in laboratory cages [34]. In subsequent experiments, there was a variety of variables including species, sex, and age, and also EE variables that contributed to the diversity of the results [35].
In the early 1960s, Will et al. [36] conducted a series of experiments to explore the effects of EE on brain development and cognitive ability; they provided a standardized model of EE conditions that is still in use today.

The classic EE definition is a complex combination of inanimate and social stimuli; in an experimental model, this is accomplished by raising a large number of animals in large groups in an environment that maintains a wide range of stimuli, including frequently changed objects (such as toys, nesting materials, and stairs). A basic component of typical EE is the opportunity to obtain a high level of voluntary physical activity on a running wheel [5]. In some experimental models, EE may also include increasing social stimulation by the addition of more animals to each cage [34]. These configurations provide richness in 3 key areas: novelty, socialization, and exercise [37].

Living in EE conditions provides animals with the optimal conditions to enhance exploration, cognitive activities, social interaction, and physical exercise [38]. This practice is thought to lead to greater physical and psychological or emotional well-being, similar to in the natural environment, and is also thought to change the physiological responses of animals and induce neurobiological changes [39].

It has been demonstrated that EE improves behavioral, cellular, and molecular defects in animal models of neurological and mental disorders, including AD, Parkinson's disease, stroke, epilepsy, Huntington's disease (HD), and schizophrenia [38]. For example, EE elevated diminished cell proliferation and neurogenesis to wildtype levels in TgCRND8 mice with AD-like pathology and upregulated the expression of plasticity-associated structural proteins in TgCRND8 mice [40]. Furthermore, in mouse models of $\mathrm{HD}$, it has been demonstrated that $\mathrm{EE}$ delays the onset and progression of motor symptoms and cognitive deficits [41]. EE can also be used in experimental models of the daily cognitive and physical activities of humans; the application of such models may expand our knowledge of experience- and training-induced plasticity [42] as well as our understanding of the mechanisms of cognitive enrichment, thereby promoting the development of noninvasive preventive interventions for age-related neurodegenerative and other diseases [43].

\section{The Effect of EE on the Brain}

In terms of anatomical structure, EE can significantly increase the thickness and weight of the cortex, the size of the cell body and nucleus, the number of dendritic 
branches, the length of dendritic spines, and the size and number of synapses [5]. It has also been suggested that EE increases the total number of granulosa cells, alters dendritic complexity and spinal density, and promotes vascularization in the hippocampus [44]. It also increases the expression of synaptophysin and postsynaptic density-95 protein, which leads to increased synaptogenesis. A study has shown that EE decreases neuronal density but increases the synapse-to-neuron ratio, synaptic disc diameter, and subsynaptic plate perforation [45]. Similar morphological changes are observed in the dentate granule neurons and pyramidal cells in the CA1 and CA3 regions of the hippocampus [46].

In addition, exposure to EE enhances synaptic transmission and plasticity, increases the survival of regenerated neurons, reduces apoptosis, and promotes the expression of molecules involved in neuronal signal transduction [47]. Plasticity refers to the ability of a system to change its constituent elements and internal connection network to achieve new functions under long-term environmental constraints [36]. Neuroplasticity is an inherent characteristic of the mammalian brain that enables it to adapt to environmental changes [48]. In a recent animal study, Harati et al. [49] showed that prolonged EE can prevent cognitive decline caused by aging in female rats.

Studies have confirmed that changes in brain structure and behavior after experiencing EE are related to changes in brain neurochemistry and physiology. Several aspects of hippocampal function are reported to be enhanced by $\mathrm{EE}$, such as LTP, neurogenesis, dendritic spine growth, neurotrophin mRNA expression, and the activation of mitogen-activated protein kinase (MAPK) and cAMP response element-binding protein (CREB) [47]. EE also increases the ability of hippocampal neurogenesis cells to integrate into functional circuits [45], which is thought to be mediated by the recruitment of vascular endothelial growth factor (VEGF) and T cells and the activation of microglia [50].

A large number of genes respond to $\mathrm{EE}$ and change their expression levels; most of them belong to functional categories related to regulating neuronal structure, synaptic transmission and plasticity, neuronal excitability, and neuroprotection [5]. EE can mediate the increased survival of newly formed neurons in the hippocampus by activating transcription factors and inducing the expression of growth factors, thereby increasing resistance to brain injury. In previous studies, it has been repeatedly demonstrated that $\mathrm{EE}$ enhances the expression of mRNA encoding trophic factors such as NGF, BDNF, NT-3, and
GDNF as well as the number of NGF proteins and the density of NGF receptors [36].

Rosenzweig et al. [51] found, for the first time, that EE can increase the activity of acetylcholinesterase (AChE), indicating that it has an effect on the cholinergic system. Subsequent studies confirmed and extended this preliminary observation to other neurotransmitter systems that have diffuse projections to the entire brain, such as the serotonergic and norepinephrine systems [5].

\section{The Influence of EE on POCD}

EE can greatly improve the symptoms of POCD. In various learning tasks, $\mathrm{EE}$ has been proven to enhance memory. The EE-exposed mice performed better on the water maze task, a spatial memory test, than the control group in standard housing. Similarly, the voluntary wheel-running and treadmill training of EE have been shown to enhance spatial learning [45]. Compared with rats kept in standard living conditions, rats kept in EE conditions showed a higher level of neuroplasticity. In the EE group, the expression of nerve growth factor in the hippocampus was increased and was positively correlated with the survival and recognition ability of precursor cells in the dentate gyrus [46].

\section{Effects of EE on Neuroinflammation and Glial Cells}

The expression of the chemokines CCL2, CCL3, and CXCL2, the TNF family, and the proinflammatory cytokine IL- $1 \beta$ is significantly decreased in EE rats [52]. IL-6 is mainly present in activated glial cells, and Griñan-Ferré et al. [53] proved that the expression of the $I L-6$ gene decreased significantly under EE conditions. EE also limited the corticosterone-induced increase in IL- 6 and TNF- $\alpha$ mRNAs in the hippocampus and hypothalamus and the increase in IL-1 $\beta$ mRNA levels in the hippocampus [54].

The results of in vitro experiments found that, after peripheral immune stimulation with lipopolysaccharide, the release of proinflammatory cytokines from microglia isolated from the aged hippocampus was higher than that from the young hippocampus. However, EE can reduce this exaggerated cytokine response, and these findings suggest that EE can prevent the development of POCD by reversing the proinflammatory phenotype of age-related microglia in the hippocampus [55]. This may be related to an increase in adiponectin (APN) in the brain induced by EE [54]. APN induces the expression of the anti-inflammatory cytokine IL-10 in human monocytes and macro- 
phages [56] and inhibits the release of IL-6 from macrophages [57]. Experiments by Chabry et al. [54] demonstrated that EE reduced the percentage of microglia expressing MHC-II and its surface TLR4/CD14 complex.

In the rodent brain, an increased number of glial cells and morphological changes in these cells have been described after EE. These morphological changes may be involved in mediating the interaction between new dendritic spines and astrocyte branches [55]. Spatial learning in a water maze contributes to the induction of basic fibroblast growth factor 2 (FGF-2), which plays a key trophic role in various types of neurons and promotes the proliferation or responsiveness of astrocytes [58]. EE can also closely regulate the concentrations of ApoE and $S 100 \beta$ in the peri-infarct region to the levels that are most conducive to glial scar formation, synaptic plasticity, and cell growth [59].

\section{Effects of EE on Ischemia and Oxidative Stress}

Briones et al. [60] found that, during rehabilitation after middle cerebral artery (MCA) occlusion (compared with the other groups), ischemic animals receiving EE rehabilitation exhibited a significantly increased degree of dendritization in the undamaged contralateral cortex. Experiments by Ruscher et al. [61] indicated that living in EE conditions could significantly reduce the increase in ApoE and IL- $1 \beta$ expression that occurs during the recovery after experimental middle cerebral artery occlusion (MCAO), and could specifically reduce the increase in reactive astrocytes around the infarcted area and at the edge of the proximal infarcted area. Yu et al. [62] found that pre-exposure to EE reduces the mRNA levels of $\mathrm{iNOS}$ and $\mathrm{nNOS}$ after MCAO, thus reducing inflammation, and that this reduction may be related to blocking the activation of ERK1/2 in microglia [63]. These studies indicate that the protective effect of $\mathrm{EE}$ on cerebral ischemia may inhibit POCD caused by CPB during cardiac surgery.

$\mathrm{EE}$ has been suggested to increase cerebral angiogenesis and reduce the expression of oxidative stress-related and apoptotic enzymes in the brain in an AD model [40]. EE may prevent the developmental loss of parvalbumin-immunoreactive cells in the prefrontal cortex induced by juvenile hypoxia and reduce neurobehavioral changes by inhibiting oxidative stress derived from NOX2 (NADPH oxidase 2). Moreover, EEsignificantly reduced the anxiety-like behaviors induced by daily intermittent hypoxia [63].

\section{The Influence of Voluntary Exercise in EE}

Studies have revealed that voluntary exercise in EE increases the thickness of the motor cortex and the expres-

POCD and the Protective Effects of

Enriched Environment: A Review sion of trophic factors such as BDNF, NGF, and FGF in the brain. Falkenberg et al. [64] found that the BDNF mRNA level in the hippocampus of rats increased and spatial memory was improved after EE. Dahlqvist et al. [65] proved that the social component of EE housing increases the expression of NGF mRNA in several cortical and hippocampal CA1 regions. Voluntary exercise also enhanced neurogenesis, related to the increase in exercise-induced IGF-1 uptake, in the hippocampus of adult animals [66]. Wadowska et al. [67] found that EE can enhance IGF-1 signaling through the Akt pathway and then reduce hippocampal neuronal loss and apoptosis after transient global cerebral ischemia. Intracerebroventricular injection of IGF also increased neurogenesis in the dentate gyrus of rats without increasing epidermal growth factor (EGF) or FGF2 [68]. Voluntary exercise can also upregulate MKP-1, which plays an important role in the negative regulation of MAPK activation in proinflammatory macrophages [69].

The voluntary wheel-running associated with EE has been shown to provide protection against ischemia. In gerbils, providing a running wheel before surgery followed by $15 \mathrm{~min}$ of global cerebral ischemia reduced the mortality rate from 55 to $10 \%$, and hippocampal cell damage was also reduced [70]. Voluntary exercise has also been shown to reduce inflammation and oxidative stress in the brain. The studies of Speisman et al. [71] on aged rats suggested that voluntary daily exercise on a running wheel reduced hippocampal IL-1 $\beta$ and serum monocyte chemoattractant protein (MCP)-1 levels.

It has been found that both short- and long-term voluntary exercise in EE affects the expression of hippocampal genes; upregulation is observed in many genes, especially those related to the glutamatergic system, while genes related to the GABA system are downregulated [36]. Segovia et al. [72] found that the level of glutamate increased in the CA3 of aged rats after EE, and this increased the excitatory synaptic potential and LTP. Moreover, the increase in IGF$1 \mathrm{R}$ phosphorylation in animals exposed to EE indicates that EE can regulate injury-induced glutamate toxicity by reducing NMDAR1 phosphorylation [67]. Voluntary exercise can also affect cholinergic systems, such as choline uptake in the hippocampus and cortex, and enhance the activity of opioid systems [73]. In addition, monoamines, such as norepinephrine and serotonin, are activated by running or physical activity [74], which in turn increases synaptic plasticity and neurogenesis [45].

\section{Effect of EE on Mechanisms Related to AD}

Studies have indicated that AD11 mice, which show progressive behavioral impairment, provided with EE be- 
fore the onset of behavioral impairment have preserved visual recognition memory and spatial memory. Longterm EE prevents the loss of cholinergic neurons in the forebrain, which may be related to the increased expression of neurotrophins, especially NGF and BDNF, in the target area of basal forebrain projections. In addition, in AD11 mice, exposure to EE reduced the presence of $A \beta$ clusters in the hippocampus [75]. Hu et al. [76] demonstrated that EE could also reduce the hyperphosphorylation of Tau protein. These studies suggest that EE can effectively reduce and prevent the occurrence of $\mathrm{AD}$ at the molecular level and that such molecular mechanisms may also act on POCD, thereby inhibiting its occurrence and development. Some studies have also evaluated the effect on elderly subjects of participating in activities with cognitive requirements and found that the activities with higher cognitive requirements reduced the risk of developing AD [77].

\section{Conclusion}

POCD is a common postoperative brain complication that greatly reduces cognitive ability, such as memory, attention, and the execution of tasks, and it has an enormous impact on human life. The occurrence and development of POCD is closely related to advanced age, invasive surgery, duration of anesthesia, and level of education, and the main pathogenesis of POCD is neuroinflammation in the brain. HMGB1, IL-1, IL-6, TNF- $\alpha$ and inflammatory chemokines can promote POCD development. Hypoxia and oxidative stress in the brain and changes in the phenotype and receptor expression of microglia and astrocytes under inflammatory conditions also promote the occurrence of POCD. On the other hand, EE can affect the release and inhibition of related factors in the brain by enhancing novelty, social contact, and physical exercise, and thus improve the cognitive ability, memory, and other functions of the brain. EE can significantly increase synaptic transmission and plasticity in the hippocampus, and it enhances the release of trophic factors such as BDNF and NGF so that the brain is more resistant to injury. EE also inhibits the release of inflammatory cytokines and chemokines in the brain, promotes the enhancement of cholinergic and glutamatergic activities and the inhibition of the GABA system, and negatively regulates the occurrence and development of POCD. These findings suggest that EE could be used as an effective preventive and therapeutic measure for POCD. However, the specific effect of EE can only be accurately determined after improving the evaluation criteria of POCD. Meanwhile, the molecular changes regulated by EE can also provide ideas for drug therapy targets. However, there are experimental shortfalls that reduce the translation of research results to humans. We should therefore retain a focus on preclinical research to promote progress in disease research. For example, we could explore the influence of the upstream targets of cytokines and chemokines, such as IGF-I or FGF-2, on the progression of POCD in EE.

\section{Acknowledgement}

The authors thank The First Affiliated Hospital of Nanchang University which instructed and supported the article.

\section{Conflict of Interest Statement}

The authors have no sponsorship or funding arrangements to disclose relating to this paper.

\section{Funding Sources}

This study was supported by a grant (No. 82060217 to J.M.) from the National Natural Science Foundation of China, Beijing, China.

\section{Author Contributions}

M.H. completed the whole work of this manuscript, including searching the literature, writing, and drawing the figure. J.M. is responsible for the revision of grammar and the improvement of sentences.

\section{References}

1 Pappa M, Theodosiadis N, Tsounis A, Sarafis P. Pathogenesis and treatment of post-operative cognitive dysfunction. Electron Physician. $2017 \mathrm{Feb}$;9(2):3768-75.

2 Kotekar N, Shenkar A, Nagaraj R. Postoperative cognitive dysfunction - current preventive strategies. Clin Interv Aging. 2018 Nov;13:2267-73.
3 Monk TG, Price CC. Postoperative cognitive disorders. Curr Opin Crit Care. 2011 Aug; 17(4):376-81

4 Xie Z, Tanzi RE. Alzheimer's disease and post-operative cognitive dysfunction. Exp Gerontol. 2006 Apr;41(4):346-59.
5 Sale A, Berardi N, Maffei L. Enrich the environment to empower the brain. Trends Neurosci. 2009 Apr;32(4):233-9.

6 Bedford PD. Adverse cerebral effects of anaesthesia on old people. Lancet. 1955 Aug; 269(6884):259-63 
7 Moller JT, Cluitmans P, Rasmussen LS, Houx P, Rasmussen H, Canet J, et al.; International Study of Post-Operative Cognitive Dysfunction. Long-term postoperative cognitive dysfunction in the elderly ISPOCD1 study. ISPOCD investigators. Lancet. 1998 Mar; 351(9106):857-61.

8 Kok WF, Koerts J, Tucha O, Scheeren TW, Absalom AR. Neuronal damage biomarkers in the identification of patients at risk of longterm postoperative cognitive dysfunction after cardiac surgery. Anaesthesia. 2017 Mar; 72(3):359-69.

9 Nemeth E, Vig K, Racz K, Koritsanszky KB, Ronkay KI, Hamvas FP, et al. Influence of the postoperative inflammatory response on cognitive decline in elderly patients undergoing on-pump cardiac surgery: a controlled, prospective observational study. BMC Anesthesiol. 2017 Aug;17(1):113.

10 Monk TG, Weldon BC, Garvan CW, Dede DE, van der Aa MT, Heilman KM, et al. Predictors of cognitive dysfunction after major noncardiac surgery. Anesthesiology. 2008 Jan;108(1):18-30.

11 Steinmetz J, Rasmussen LS. Peri-operative cognitive dysfunction and protection. Anaesthesia. 2016 Jan;71 Suppl 1:58-63.

12 Wang W, Wang Y, Wu H, Lei L, Xu S, Shen X, et al. Postoperative cognitive dysfunction: current developments in mechanism and prevention. Med Sci Monit. 2014 Oct;20:1908-12.

13 Berger M, Nadler JW, Browndyke J, Terrando $\mathrm{N}$, Ponnusamy V, Cohen HJ, et al. Postoperative Cognitive Dysfunction: Minding the Gaps in Our Knowledge of a Common Postoperative Complication in the Elderly. Anesthesiol Clin. 2015 Sep;33(3):517-50.

14 Czyż-Szypenbejl K, Mędrzycka-Dąbrowska W, Kwiecień-Jaguś K, Lewandowska K. The Occurrence of Postoperative Cognitive Dysfunction (POCD) - Systematic Review. Psychiatr Pol. 2019 Feb;53(1):145-60.

15 Wollman SB, Orkin LR. Postoperative human reaction time and hypocarbia during anaesthesia. Br J Anaesth. 1968 Dec;40(12):920-6.

16 Salameh A, Dhein S, Dähnert I, Klein N. Neuroprotective Strategies during Cardiac Surgery with Cardiopulmonary Bypass. Int J Mol Sci. 2016 Nov; 17(11):1945.

17 Cibelli M, Fidalgo AR, Terrando N, Ma D, Monaco C, Feldmann M, et al. Role of interleukin-1beta in postoperative cognitive dysfunction. Ann Neurol. 2010 Sep;68(3):360-8.

18 Lin D, Zuo Z. Isoflurane induces hippocampal cell injury and cognitive impairments in adult rats. Neuropharmacology. 2011 Dec; 61(8):1354-9.

19 Ito A, Goto T, Maekawa K, Baba T, Mishima Y, Ushijima K. Postoperative neurological complications and risk factors for pre-existing silent brain infarction in elderly patients undergoing coronary artery bypass grafting. J Anesth. 2012 Jun;26(3):405-11.

20 Skvarc DR, Berk M, Byrne LK, Dean OM, Dodd S, Lewis M, et al. Post-Operative Cognitive Dysfunction: an exploration of the in- flammatory hypothesis and novel therapies. Neurosci Biobehav Rev. 2018 Jan;84:116-33.

21 Terrando N, Eriksson LI, Ryu JK, Yang T, Monaco C, Feldmann M, et al. Resolving postoperative neuroinflammation and cognitive decline. Ann Neurol. 2011 Dec;70(6): 986-95.

22 Awooda HA, Lutfi MF, Sharara GM, Saeed AM. Role of N-Nitro-L-Arginine-Methylester as anti-oxidant in transient cerebral ischemia and reperfusion in rats. Exp Transl Stroke Med. 2013 Jan;5(1):1.

23 Kim ID, Lee JK. HMGB1-Binding Heptamer Confers Anti-Inflammatory Effects in Primary Microglia Culture. Exp Neurobiol. 2013 Dec;22(4):301-7.

24 Zhang T, Tian X, Wang Q, Tong Y, Wang H, $\mathrm{Li} Z$, et al. Surgical stress induced depressive and anxiety like behavior are improved by dapsone via modulating NADPH oxidase level. Neurosci Lett. 2015 Jan;585:103-8.

25 Sorce S, Krause KH. NOX enzymes in the central nervous system: from signaling to disease. Antioxid Redox Signal. 2009 Oct;11(10): 2481-504.

26 Androsova G, Krause R, Winterer G, Schneider R. Biomarkers of postoperative delirium and cognitive dysfunction. Front Aging Neurosci. 2015 Jun; 7:112.

27 Jiang J, Jiang H. Effect of the inhaled anesthetics isoflurane, sevoflurane and desflurane on the neuropathogenesis of Alzheimer's disease (review). Mol Med Rep. 2015 Jul;12(1):3-12.

28 Eckenhoff RG, Johansson JS, Wei H, Carnini A, Kang B, Wei W, et al. Inhaled anesthetic enhancement of amyloid-beta oligomerization and cytotoxicity. Anesthesiology. 2004 Sep;101(3):703-9.

29 Xie Z, Culley DJ, Dong Y, Zhang G, Zhang B, Moir RD, et al. The common inhalation anesthetic isoflurane induces caspase activation and increases amyloid beta-protein level in vivo. Ann Neurol. 2008 Dec;64(6):618-27.

30 Pepe MG, Curtiss LK. Apolipoprotein E is a biologically active constituent of the normal immunoregulatory lipoprotein, LDL-In. J Immunol. 1986 May;136(10):3716-23.

31 Aleong R, Blain JF, Poirier J. Pro-inflammatory cytokines modulate glial apolipoprotein E secretion. Curr Alzheimer Res. 2008 Feb; 5(1):33-7.

32 Zurek AA, Yu J, Wang DS, Haffey SC, Bridgwater EM, Penna A, et al. Sustained increase in a5GABAA receptor function impairs memory after anesthesia. J Clin Invest. 2014 Dec;124(12):5437-41.

33 Hebb DO. The effects of early experience on problem solving at maturity. Am Psychol. 1947;2:306-7.

34 Nithianantharajah J, Hannan AJ. Enriched environments, experience-dependent plasticity and disorders of the nervous system. Nat Rev Neurosci. 2006 Sep;7(9):697-709.

35 Ohline SM, Abraham WC. Environmental enrichment effects on synaptic and cellular physiology of hippocampal neurons. Neuropharmacology. 2019;145(Pt A):3-12.
36 Will B, Galani R, Kelche C, Rosenzweig MR Recovery from brain injury in animals: relative efficacy of environmental enrichment, physical exercise or formal training (19902002). Prog Neurobiol. 2004 Feb;72(3):16782.

37 Crofton EJ, Zhang Y, Green TA. Inoculation stress hypothesis of environmental enrichment. Neurosci Biobehav Rev. 2015 Feb;49: 19-31.

38 Hannan AJ. Environmental enrichment and brain repair: harnessing the therapeutic effects of cognitive stimulation and physical activity to enhance experience-dependent plasticity. Neuropathol Appl Neurobiol. 2014 Feb;40(1):13-25.

39 Girbovan C, Plamondon H. Environmental enrichment in female rodents: considerations in the effects on behavior and biochemical markers. Behav Brain Res. 2013 Sep;253:17890.

40 Herring A, Ambrée O, Tomm M, Habermann H, Sachser N, Paulus W, et al. Environmental enrichment enhances cellular plasticity in transgenic mice with Alzheimer-like pathology. Exp Neurol. 2009 Mar;216(1):184-92.

41 Laviola G, Hannan AJ, Macrì S, Solinas M, Jaber M. Effects of enriched environment on animal models of neurodegenerative diseases and psychiatric disorders. Neurobiol Dis. 2008 Aug;31(2):159-68

42 Mirochnic S, Wolf S, Staufenbiel M, Kempermann G. Age effects on the regulation of adult hippocampal neurogenesis by physical activity and environmental enrichment in the APP23 mouse model of Alzheimer disease. Hippocampus. 2009 Oct;19(10):1008-18.

43 Redolat R, Mesa-Gresa P. Potential benefits and limitations of enriched environments and cognitive activity on age-related behavioural decline. Curr Top Behav Neurosci. 2012;10: 293-316.

44 Bekinschtein P, Oomen CA, Saksida LM, Bussey TJ. Effects of environmental enrichment and voluntary exercise on neurogenesis, learning and memory, and pattern separation: BDNF as a critical variable? Semin Cell Dev Biol. 2011 Jul;22(5):536-42.

45 van Praag H, Kempermann G, Gage FH. Neural consequences of environmental enrichment. Nat Rev Neurosci. 2000 Dec;1(3): 191-8.

46 Rampon C, Tang YP, Goodhouse J, Shimizu E, Kyin M, Tsien JZ. Enrichment induces structural changes and recovery from nonspatial memory deficits in CA1 NMDAR1knockout mice. Nat Neurosci. 2000 Mar;3(3): 238-44.

47 Petrosini L, De Bartolo P, Foti F, Gelfo F, Cutuli D, Leggio MG, et al. On whether the environmental enrichment may provide $\operatorname{cog}$ nitive and brain reserves. Brain Res Brain Res Rev. 2009 Oct;61(2):221-39.

48 Nilsson M, Pekny M. Enriched environment and astrocytes in central nervous system regeneration. J Rehabil Med. 2007 May;39(5): 345-52.
POCD and the Protective Effects of Enriched Environment: A Review
Neurodegener Dis 2020;20:113-122 DOI: $10.1159 / 000513196$ 
49 Harati H, Barbelivien A, Herbeaux K, Muller MA, Engeln M, Kelche C, et al. Lifelong environmental enrichment in rats: impact on emotional behavior, spatial memory vividness, and cholinergic neurons over the lifespan. Age (Dordr). 2013 Aug;35(4):1027-43.

50 During MJ, Cao L. VEGF, a mediator of the effect of experience on hippocampal neurogenesis. Curr Alzheimer Res. 2006 Feb;3(1): 29-33.

51 Rosenzweig MR, Bennett EL, Diamond MC. Effects of differential environments on brain anatomy and brain chemistry. Proc Annu Meet Am Psychopathol Assoc. 1967;56:4556.

52 Williamson LL, Chao A, Bilbo SD. Environmental enrichment alters glial antigen expression and neuroimmune function in the adult rat hippocampus. Brain Behav Immun. 2012 Mar;26(3):500-10.

53 Griñan-Ferré C, Pérez-Cáceres D, GutiérrezZetina SM, Camins A, Palomera-Avalos V, Ortuño-Sahagún D, et al. Environmental Enrichment Improves Behavior, Cognition, and Brain Functional Markers in Young Senescence-Accelerated Prone Mice (SAMP8). Mol Neurobiol. 2016 May;53(4):2435-50.

54 Chabry J, Nicolas S, Cazareth J, Murris E, Guyon A, Glaichenhaus N, et al. Enriched environment decreases microglia and brain macrophages inflammatory phenotypes through adiponectin-dependent mechanisms: relevance to depressive-like behavior. Brain Behav Immun. 2015 Nov;50:275-87.

55 Kawano T, Eguchi S, Iwata H, Tamura T, Kumagai N, Yokoyama M. Impact of Preoperative Environmental Enrichment on Prevention of Development of Cognitive Impairment following Abdominal Surgery in a Rat Model. Anesthesiology. 2015 Jul;123(1):160 70.

56 Weigert J, Neumeier M, Wanninger J, Wurm S, Kopp A, Schober F, et al. Reduced response to adiponectin and lower abundance of adiponectin receptor proteins in type 2 diabetic monocytes. FEBS Lett. 2008 May;582(12): 1777-82.

57 Wulster-Radcliffe MC, Ajuwon KM, Wang J, Christian JA, Spurlock ME. Adiponectin differentially regulates cytokines in porcine macrophages. Biochem Biophys Res Commun. 2004 Apr;316(3):924-9.
58 Gómez-Pinilla F, So V, Kesslak JP. Spatial learning and physical activity contribute to the induction of fibroblast growth factor: neural substrates for increased cognition associated with exercise. Neuroscience. $1998 \mathrm{Jul}$ 85(1):53-61.

59 Selinfreund RH, Barger SW, Pledger WJ, Van Eldik LJ. Neurotrophic protein S100 beta stimulates glial cell proliferation. Proc Natl Acad Sci USA. 1991 May;88(9):3554-8.

60 Briones TL, Therrien B, Metzger B. Effects of environment on enhancing functional plasticity following cerebral ischemia. Biol Res Nurs. 2000 Apr;1(4):299-309.

61 Ruscher K, Johannesson E, Brugiere E, Erickson A, Rickhag M, Wieloch T. Enriched environment reduces apolipoprotein $\mathrm{E}$ (ApoE) in reactive astrocytes and attenuates inflammation of the peri-infarct tissue after experimental stroke. J Cereb Blood Flow Metab. 2009 Nov;29(11):1796-805.

62 Yu K, Wu Y, Hu Y, Zhang Q, Xie H, Liu G, et al. Neuroprotective effects of prior exposure to enriched environment on cerebral ischemia/reperfusion injury in rats: the possible molecular mechanism. Brain Res. 2013 Nov; 1538:93-103.

63 Yoo BK, Choi JW, Han BH, Kim WK, Kim $\mathrm{HC}$, Ko KH. Role of MAPK/ERK1/2 in the glucose deprivation-induced death in immunostimulated astroglia. Neurosci Lett. 2005 Mar;376(3):171-6.

64 Falkenberg T, Mohammed AK, Henriksson B, Persson H, Winblad B, Lindefors N. Increased expression of brain-derived neurotrophic factor mRNA in rat hippocampus is associated with improved spatial memory and enriched environment. Neurosci Lett. 1992 Apr;138(1):153-6.

65 Dahlqvist P, Rönnbäck A, Risedal A, Nergårdh R, Johansson IM, Seckl JR, et al. Effects of postischemic environment on transcription factor and serotonin receptor expression after permanent focal cortical ischemia in rats. Neuroscience. 2003;119(3):643-52.

66 Carro E, Nuñez A, Busiguina S, Torres-Aleman I. Circulating insulin-like growth factor I mediates effects of exercise on the brain. J Neurosci. 2000 Apr;20(8):2926-33.

67 Wadowska M, Woods J, Rogozinska M, Briones TL. Neuroprotective effects of enriched environment housing after transient global cerebral ischaemia are associated with the upregulation of insulin-like growth factor-1 signalling. Neuropathol Appl Neurobiol. 2015 Jun;41(4):544-56.
68 Aberg MA, Aberg ND, Hedbäcker H, Oscarsson J, Eriksson PS. Peripheral infusion of IGFI selectively induces neurogenesis in the adult rat hippocampus. J Neurosci. 2000 Apr;20(8): 2896-903.

69 Eyre HA, Papps E, Baune BT. Treating depression and depression-like behavior with physical activity: an immune perspective. Front Psychiatry. 2013 Feb;4:3.

70 Stummer W, Baethmann A, Murr R, Schürer L, Kempski OS. Cerebral protection against ischemia by locomotor activity in gerbils. Underlying mechanisms. Stroke. 1995 Aug; 26(8):1423-9.

71 Speisman RB, Kumar A, Rani A, Foster TC, Ormerod BK. Daily exercise improves memory, stimulates hippocampal neurogenesis and modulates immune and neuroimmune cytokines in aging rats. Brain Behav Immun. $2013 \mathrm{Feb} ; 28: 25-43$

72 Segovia G, Yagüe AG, García-Verdugo JM, Mora F. Environmental enrichment promotes neurogenesis and changes the extracellular concentrations of glutamate and GABA in the hippocampus of aged rats. Brain Res Bull. 2006 Jun; 70(1):8-14.

73 Fordyce DE, Farrar RP. Physical activity effects on hippocampal and parietal cortical cholinergic function and spatial learning in F344 rats. Behav Brain Res. 1991 May;43(2): $115-23$.

74 Chaouloff F. Physical exercise and brain monoamines: a review. Acta Physiol Scand. 1989 Sep;137(1):1-13.

75 Berardi N, Braschi C, Capsoni S, Cattaneo A, Maffei L. Environmental enrichment delays the onset of memory deficits and reduces neuropathological hallmarks in a mouse model of Alzheimer-like neurodegeneration. J Alzheimers Dis. 2007 Jun;11(3):359-70.

$76 \mathrm{Hu}$ YS, Xu P, Pigino G, Brady ST, Larson J, Lazarov O. Complex environment experience rescues impaired neurogenesis, enhances synaptic plasticity, and attenuates neuropathology in familial Alzheimer's disease-linked APPswe/PS1DeltaE9 mice. FASEB J. 2010 Jun;24(6):1667-81.

77 Wilson RS, Bennett DA, Bienias JL, Aggarwal NT, Mendes De Leon CF, Morris MC, et al. Cognitive activity and incident $\mathrm{AD}$ in a population-based sample of older persons. Neurology. 2002 Dec;59(12):1910-4. 